\title{
Time Perception: Does it Distinguish ADHD and RD Children in a Clinical Sample?
}

\author{
R. McGee,,$^{1,2,5}$ D. Brodeur, ${ }^{2}$ D. Symons, ${ }^{2}$ B. Andrade, ${ }^{3}$ and C. Fahie ${ }^{4}$
}

Received September 25, 2003; revision received March 26, 2004; accepted April 8, 2004

This study used a double-dissociation design to evaluate whether children with ADHD demonstrated specific deficits relative to children with Reading Disorders. Recent theory suggests that ADHD children have deficits in time perception and working memory, whereas RD children have deficits in phonological decoding. The performance of 113 clinic-referred children aged 6-11 was examined using measures of working memory, phonological processing, and time perception. Respondents completed two time production tasks in which they were to judge when 30-s had elapsed, and another in which they were asked to estimate the duration of the Conners' CPT (CCPT). Time Perception and phonological processing variables were submitted to a $2 \times 2$ ANCOVA (ADHD vs. RD), covarying for age, SES, IQ, and working memory. Children with ADHD were more likely to overestimate the time taken for the CCPT than children without ADHD, but no group differences were found on the 30-s estimation tasks. Children with RD did not display deficits in time estimation, but showed deficits in auditory phonological processing. The lack of interaction effects supported an "etiological subtype" over the "phenocopy" model of ADHD and RD. No group differences were detected using the CCPT. Although our previous studies did not find an order effect for the Conners' CPT in a 1-hr battery, a fatigue effect was evident with a 1.5-hr battery. The implications for Barkley's behavioral inhibition theories (R. Barkley, 1997) are discussed.

KEY WORDS: attention deficit hyperactivity disorder; assessment; time perception; continuous performance test; reading disorder.

In clinical practice, many children are referred for inattention at home and school. Clinicians must distinguish those children with Attention Deficit Hyperactivity Disorder (ADHD) from those with other clinical conditions that also manifest as inattention. In particular, it is important to distinguish children with Reading Disorders (RD) from those with ADHD, given that failure to read can

\footnotetext{
${ }^{1}$ Child and Adolescent Mental Health Services, Valley Regional Hospital, Kentville, Nova Scotia, Canada.

${ }^{2}$ Department of Psychology, Acadia University, Wolfville, Nova Scotia, Canada.

${ }^{3}$ Department of Psychology, Dalhousie University, Halifax, Nova Scotia, Canada.

${ }^{4}$ Izaak Walton Killam Hospital for Children, Halifax, Nova Scotia, Canada.

${ }^{5}$ Address all correspondence to: Robin McGee, Ph.D., Child and Adolescent Mental Health Services, Valley Regional Hospital, 150 Exhibition Street, Kentville, Nova Scotia, Canada B4N 5E3; e-mail: robinmcgee@ accessujave.ca.
}

appear as inattention and distraction at school. Children with ADHD and RD are thought to share similar deficits in rapid scanning, rapid naming, general reading fluency, and slower processing speed (Barkley, Grodzinsky, \& DuPaul, 1992). It is well-established that ADHD often co-occurs with RD (August \& Garfinkel, 1990), and comorbidity of RD and ADHD is estimated at approximately 23\% of children with ADHD (Semrud-Clikeman et al., 1992). Although research has identified measures that discriminate children with RD and ADHD from nonclinical controls (Barkley, 1991; Shapiro \& Herod, 1994), few studies have found brief instruments that reliably distinguish them from clinical controls or from each other. Ideally, research on group differences can lead eventually to improved diagnostic standards. Individual diagnostic accuracy is important, because there are different evidencebased treatment implications for each diagnosis: stimulant medication and behavior therapy for ADHD, and phonics instruction for RD. 
This study examined group differences in cognitive processing of ADHD and RD children using a doubledissociation (DD) design. Pennington, Grossier, and Welsh (1993) have described DD designs as those in which "pure" forms of two disorders are contrasted with a comorbid group, as well as a control group. DD designs allow for some inferences regarding the causal underpinnings of each disorder. The two disorders should exhibit opposite profiles on two contrasting cognitive domains, each of which is hypothesized to be central to one disorder and not the other. The "phenocopy" hypothesis (one disorder is primary, the other is secondary) predicts that the comorbid group's profile will be similar to one of the pure groups. The "etiological subtype" hypothesis (the disorders are etiologically distinct) predicts that the comorbid group will show additive effects of the two disorders.

Several studies have employed DD designs contrasting ADHD and RD children (Breier, Gray, Fletcher, Foorman, \& Klaas, 2002; Felton, Wood, Brown, Campbell, \& Harter, 1987; McGee, Clark \& Symons, 2000; Pennington et al., 1993; Purvis \& Tannock, 2000; Seidman, Biederman, Monuteaux, Doyle, \& Faraone, 2001; Willcutt et al., 2001). Although these studies have employed a $2 \times 2$ (ADHD vs. RD) design, methodology has varied. Studies differ with respect to the definition and diagnosis of the two disorders. Whereas some have used IQ-achievement discrepancy scores to define the RD group (e.g., Pennington et al., 1993), others have used cutoffs on reading achievement measures (Purvis \& Tannock, 2000). To define the ADHD groups, some researchers have used behavior rating scale cutoffs (e.g., Pennington et al., 1993), some have used DSM criteria (Willcutt et al., 2001), and some have used a combination of these approaches (e.g., McGee et al., 2000). The criteria or dependent variables have also been diverse, and control group selection has differed. Some researchers have used nonclinical controls, whereas others have used clinic-referred controls. Of those using clinical controls, some researchers have deliberately excluded affective disorders, severe family relational problems, or anxiety disorders from the control group (e.g., Purvis \& Tannock, 2000). Others have used clinic-referred children and have made no efforts to "normalize" the control group (e.g., Cohen et al., 2000; McGee et al., 2000). Studies have differed in their use of covariates or matching variables, which have included age (e.g., McGee et al., 2000), IQ scores (Felton et al., 1987; Pennington et al., 1993), and socioeconomic status (McGee et al., 2000; Seidman et al., 2001).

Despite these method differences, a ubiquitous finding has emerged consistently across most RD versus ADHD DD design studies. Children with RD are consis- tently and reliably distinguished from children without RD using measures of phonological processing (Breier et al., 2002; Felton et al., 1987; McGee et al., 2000; Pennington et al., 1993; Purvis \& Tannock, 2000; Willcutt et al., 2001). Considerable research suggests that a deficit in phonological awareness is the central feature of RD (Catts, 1989; McBride-Chang, 1995).

Far less consistent findings have been found when attempting to differentiate children with ADHD from children with RD or clinical controls. Continuous performance tasks can distinguish children with ADHD from normal children (Losier, McGrath, \& Klein, 1996). However, studies involving clinical controls indicate that continuous performance tests do not reliably distinguish children with ADHD from other clinical groups (Barkley, DuPaul, \& McMurray, 1990; Corkum \& Siegel, 1993; Koriath, Gualtieri, Van Bourgondien, Quade, \& Werry, 1985). In addition, children with RD tend to perform poorly on continuous performance tests (Eliason \& Richman, 1987; Tarnowski, Prinz, \& Nay, 1986). Two DD studies have used the commercially available Conners' Continuous Performance Test (CCPT; Conners, 1995). McGee et al. (2000) found that children with RD did more poorly on the CCPT relative to children with ADHD, obtaining higher scores on the overall index and having greater problems with errors and reaction times. The authors attributed this finding to the fact that the CCPT requires the rapid identification of letters-a known deficit in children with RD (Compton, DeFries, \& Olson, 2001). Purvis and Tannock (2000) found that children with ADHD were slower, more variable, and more error-prone on the CCPT relative to RD children, but that these results were not consistent across other inhibition tasks. The potential of the Conners' CPT for differential diagnosis has not been clearly established.

What other tasks might distinguish ADHD from RD youngsters? Recent theory regarding the core deficit of ADHD may provide ideas. Barkley (1997a, 1997b, 1997c) has espoused a new conceptualization of ADHD in which the central deficiency in ADHD is impaired behavioral inhibition. This impairment is related to and necessary for the four executive functions that subserve self-regulation. Key cognitive domains will be compromised by this core deficit: working memory and sense of time, internalization of speech, affect regulation, and "reconstitution" or the formation of novel, complex behavioral sequences. This model predicts that ADHD children "manifest an impairment in the development of sense of time and its associated retrospective and prospective functions... as a consequence of poor behavioral inhibition" (Barkley, Koplowitz, Anderson, \& McMurray, 1997, p. 359). Children with ADHD ought to be less accurate in judgements 
about time: how long something will take, and how long something did take.

Research is amassing that supports Barkley's model. Working memory deficits are well-documented in ADHD (Pennington \& Ozonoff, 1996; Stevens, Quittner, Zuckerman, \& Moore, 2002; Tannock, Ickowicz, \& Schachar, 1995). Also, recent research has explored time perception deficits of children with ADHD relative to normal controls (Barkley et al., 1997; Barkley, Edwards, Laneri, Fletcher, \& Metevia, 2001; Castellanos \& Tannock, 2002; Kerns, McInerney, \& Wilde, 2001; Kerns \& Price, 2001; West et al., 2000). Time perception deficits are found among ADHD children even when matching for working memory (Smith, Taylor, Rogers, Newman, \& Rubia, 2002). However, working memory deficits are also welldocumented in RD children (Cohen et al., 2000; Korkman \& Pesonen, 1994; Rucklidge \& Tannock, 2002; Willcutt et al., 2001). Therefore, our study contrasted RD and ADHD groups on time duration estimates controlling for working memory.

Previous studies of time perception in children with ADHD have examined perception of very brief time intervals, often in milliseconds or seconds (e.g., Smith et al., 2002; West et al., 2000). The current study sought to evaluate time perception deficits with more naturalistic tasks, comparable to those one might find in a classroom or home setting. A parent or teacher might say "get this done in 30 seconds" or "how long did that task take?" We used two conventional methods of time perception. One method set involved a time production task in which the participant was verbally told the length of time to be produced (30 s) and then was required to produce it by indicating the end of the duration. Because distracting events have been found to decrease the accuracy of children's time estimations (Barkley et al., 1997; Zakay, 1992), we manipulated the activity demands of this time production task. A second method involved a time estimation task in which the child was exposed to a specific temporal duration, and had to report verbally the perceived duration. To do this, children estimated the duration of the Conners' CPT once it was completed. Thus, we examined both a prospective time judgement and a retrospective time judgement.

This study used a DD design to evaluate the specificity of certain tasks to the differential diagnosis of ADHD versus RD. It was predicted that time perception deficits would accrue to ADHD, whereas auditory phonological problems would accrue to RD. Participants were all children consecutively referred for assessment of ADHD during a 2-year period to an outpatient mental health clinic (thus none had previous intervention for their difficulties). Use of clinical controls permits more accurate compar- isons between groups and equality for the factors that commonly contribute to clinical case status (i.e., SES, family dysfunction; Achenbach, 1991a, 1991b). No attempts were made to screen the control group to exclude other psychiatric or behavior problems.

The following hypotheses were tested:

(1) The Conners' CPT will distinguish ADHD children from RD children and clinical controls.

(2) Time perception tasks will distinguish ADHD from RD and control children, covarying for age, IQ, SES, and working memory.

(3) RD children will perform more poorly than ADHD and control children on measures of auditory phonological awareness, even when controlling for age, IQ, SES, and working memory.

(4) The comorbid group (ADHD + RD) will show additive deficits relative to the "pure" diagnostic groups on the criterion measures. No interaction effects will be found.

\section{METHOD}

\section{Participants}

Participants were 113 children ages 6-11 years ( $M=8.8, S D=1.68)$ who were consecutively referred over a 2-year period for assessment of potential ADHD to a child and adolescent outpatient mental health clinic. The age parameter was based on the normative requirements of the measures, as well as developmental research that suggests children can make time estimates reliably only after age five (Droit-Volet \& Rattat, 1999). The sample was predominantly male $(n=93,82 \%)$. The children were referred by their parents, family physicians, or school personnel. Written consent to participate in the study was obtained in each case from the parent. Because this was a prospective study, none of the children had an established diagnosis at the time of the assessment, nor were on psychoactive medication.

Children in the ADHD group met each of three criteria. Only cases that met all three criteria were defined as ADHD. If all three ADHD criteria were not met for an individual, that case was omitted from the study.

(1) Each child met the criteria for ADHD according to $D S M-I V$, as assessed by a multidisciplinary team. Team assessments involved interviews with the child, parents, and collateral sources (e.g., teachers). The medical, developmental, personal history, and DSM-IV checklist interviews described in Barkley (1997d) guided 
interviews. Team assessments also involved interpretation of behavioral rating scales.

(2) Each child was beyond clinical cutoffs on both parent and teacher ratings. The parent and teacher forms of the Conners' Rating ScaleRevised: Long Version (CPRS-R:L, CTRS-R:L, Conners, 1997) were completed. Parents also completed the Child Behavior Checklist (CBCL, Achenbach, 1991a), and teachers completed the Teacher Report Form (TRF, Achenbach, 1991b). To be defined as ADHD, each child scored over 2.0 standard deviations above the norm $(T>70)$ on the CPRS-R:L Hyperactivity subscale or the CBCL Inattention scale, and either the CTRS$\mathrm{R}: \mathrm{L}$ Hyperactivity scale or the TRF Inattention scale. If any of the four behavioral rating scales were missing because they were not returned, that child was omitted from the study.

(3) Each case file was reviewed by an independent clinical psychologist. This diagnostician checked whether each case met DSM-IV criteria for ADHD, including onset before age seven.

This three-pronged approach was used for several reasons. The Conners' and Achenbach scales are of well-accepted validity and reliability, and both are regarded as standard in ADHD diagnosis (Barkley, 1991). The statistical criterion allowed for replication of past studies (e.g., Fischer, Newby, \& Gordon, 1995) and allows for replication in future studies. Stringent cutoffs ensure a clear diagnosis. A blind check helps ensure diagnostic consistency across all cases. Using these criteria, $38 \%$ of the sample $(n=43)$ was defined as having ADHD. Of the ADHD children, few $(6 \%)$ were diagnosed as Inattentive Type; all others (93\%) met criteria for Combined Type.

Considerable research has demonstrated that the central deficit in the RD is phonological ability (McBrideChang, 1995). Specifically, reading decoding is considered a fundamental deficit. Previous studies have used reading achievement or word recognition tasks as measures of RD. In this study, we used the Word Attack subscale of the Woodcock-Johnson Revised (WJR; Woodcock \& Johnson, 1990) to define RD. Visual phonetic reading of nonsense words is regarded as a purer form of phonological awareness than word recognition, which can be influenced by sight vocabulary memorization (McBride-Chang, 1995). Moreover, because it is a visual reading task, Word Attack does not tax auditory working memory (allowing us to better evaluate the separate impact of working memory on criterion variables). The specificity of word attack skills to RD children has been shown using DD designs (Purvis \& Tannock, 2000). In this study, children were defined as RD if they had a standard score of 80 or less on WJR Word Attack. Children scoring below the selected cutoff were below the 10th percentile relative to the WJR normative sample (1.3 SD below the standard score mean of 100). Using this criterion, $39.8 \%$ of the sample $(n=45)$ were considered RD. Comorbidity of $\mathrm{ADHD}$ and $\mathrm{RD}$ was $15 \%(n=17)$.

The remainder of the sample $(37 \%, n=42)$ comprised the clinical control group. These children were given diagnoses by a multidisciplinary team using DSM-IV criteria. The diagnoses were cross-checked by an independent psychologist diagnostician. No member of the clinical control group met any of the three criteria for ADHD listed above; if they did, they were omitted from the study. The diagnoses are presented here for the purposes of sample description. Of the controls, $31 \%$ were considered to have family relational problems as their primary difficulty. Approximately 5\% were diagnosed with anxiety disorders. Another $43 \%$ were deemed to have behavior disorders other than ADHD (5\% Oppositional-defiant Disorder, 38\% Disruptive Behavior Disorder NOS). Finally, $22 \%$ were considered to have school adjustment difficulties. No statistical methods were used to create distance between the RD or ADHD children and the clinical controls: if a control child barely missed the cutoff on the $\mathrm{ADHD}$ or RD measures, he or she was retained in the clinical control condition.

\section{Measures}

\section{Covariate Measures}

Age in months was employed as a covariate due to its developmental significance (Droit-Volet \& Rattat, 1999), as well as its history as a covariate in other research using DD designs (e.g., Felton et al., 1987).

Socioeconomic Status (SES) was determined using the Blishen scales (Blishen, Carroll, \& Moore, 1987). The occupation of the primary provider was quantified. SES was a variable of focus in view of its relevance to overall adjustment, as well as its previous use in other ADHD research (e.g., Landau, Gross-Tsur, Auerbach, Van der Meere, \& Shalev, 1999; McGee et al., 2000).

Peabody Picture Vocabulary Test-Third Edition (PPVT-3; Dunn \& Dunn, 1987) is a measure of receptive vocabulary. It has been used as a proxy measure of verbal IQ and as a covariate in ADHD and RD research (e.g., Brodeur \& Pond, 2001; Fischer et al., 1995). Also, it was used to exclude subjects with severe language impairment or mental retardation from the study sample, by excluding those with PPVT-3 standard scores less than 80 (c.f., Brodeur \& Pond, 2001). 
Numbers Reversed is a subtest from the WoodcockJohnson-Revised (Woodcock \& Johnson, 1990). It is a measure of short-term auditory "working" memory. The child is required to repeat a series of random digits (after hearing them on audiotape), but must say them in backward sequence. It was selected as a covariate because it has been found to be a deficit in both ADHD and RD populations. Also, because it is an auditory task, it is suitable for partialing out same-method variance from dependent measures that are administered the same way (i.e., via headphones, requiring a verbal response). Standard scores were used.

\section{Criterion Measures}

The Conners' Continuous Performance Test (CPT Version 3.0; Conners, 1995) is a visual vigilance task that requires the child to respond to the computer screen by pressing the spacebar for every letter presented except the letter X. The test takes 14 min to complete, during which the number of omission and commission errors, reaction time, and variability of reaction times are calculated. The Conners' CPT provides an overall index to indicate attention problems, derived from a weighted regression equation of variables relevant to reaction time, omission errors, and variability of responses. Children who receive an overall index score greater than 11 can be said to have "failed" the CPT. In our study, all participants underwent the 2-min standard practice before starting the Conners' CPT. The examiner remained in the room during the administration.

Sound Blending is a subtest of the WJR. The child is must identify words in which the phonemes are separated by a 1-s interval (e.g. "st... op"). Words are presented by audiotape. This subtest evaluates the ability to recognize and integrate phonemes. Sound blending is an auditory task that requires more auditory working memory than the visual reading tasks typically used in other RD studies. Standard scores were used.

Thirty-s time estimates were time production tasks used as measures of naturalistic time perception. On the Passive task, children were asked to tell the examiner when 30 s had elapsed. On the Active task, children were instructed to rapidly complete a pegboard task, and to tell the examiner when $30 \mathrm{~s}$ had elapsed. For both tasks, the time the child took to respond was timed with a stopwatch, and the estimate recorded in seconds.

CCPT duration estimate involved a retrospective time judgment. Once the child had completed the CCPT, he or she was asked "how long did that take?". The CCPT has a standard duration of $14 \mathrm{~min}$.

\section{Procedure}

All children completed the testing measures during a 1.5-hr session with a research assistant (i.e., a Masterslevel graduate student in psychology). Measures were divided into blocks, and these blocks were administered in counterbalanced order. The cognitive tests formed a block: PPVT-3, WJR Sound Blending, WJR Word Attack, and WJR Numbers Reversed subtests. The 30-s time estimation tasks were administered as a block, with the passive estimate first. The CCPT was randomly assigned to either first or last position in the entire test battery, to test and control for fatigue effects. Qualitative behavioral observations were recorded in note form by the examiner.

\section{RESULTS}

The study design consisted of two factors: ADHD versus non-ADHD, and $\mathrm{RD}$ versus non-RD. Therefore, four groups resulted: $\mathrm{ADHD}, \mathrm{RD}, \mathrm{ADHD}$ and $\mathrm{RD}$, and clinical controls. The groups did not differ on age, gender distribution, PPVT-3 scores, or SES. Mean SES for the sample was $36.8(S D=11.3)$, which represents occupations in unskilled labor. Group differences were found on working memory scores, $F(1,109)=4.67, p<.05$. Post hoc tests revealed that WJR Numbers Reversed scores were lower among the $\mathrm{RD}$ groups $(M=93.0, S D=12.6)$ than the non-RD groups $(M=99.7, S D=16.2), t(108)=$ $2.43, p=.017$. Table I presents the means and standard deviations of covariates and dependent variables for each of the four groups. The size and gender distribution of each cell is also indicated.

\section{Correlates}

To evaluate their shared variance, we examined the correlations between cognitive variables and demographic variables within the overall sample. Active and Passive 30 -s time estimates were correlated with each other $(r=.52, p<.001)$. Age was correlated with both Active $(r=-.40, p<.0001)$, but less strongly with Passive time estimates $(r=-.24, p<.05)$. The 30 -s time estimates decreased as age increased. That is, the younger the child, the longer time elapsed before the child declared that " 30 seconds" had elapsed. There was no association between time estimates for the 30-s tasks and the estimate of the duration of the CCPT. PPVT-3 scores were marginally correlated with SES $(r=.22, p<.05)$, and Sound Blending $(r=.29, p<.01)$. As predicted from the WJR normative sample, the WJR subtests were correlated 
Table I. Means and Standard Deviations of Measures Across Groups

\begin{tabular}{|c|c|c|c|c|c|c|}
\hline \multirow[b]{2}{*}{ Variable } & \multicolumn{4}{|c|}{$M(S D)$} & \multirow[b]{2}{*}{$F$ with covariates ${ }^{a}$} & \multirow[b]{2}{*}{$F$ without covariates ${ }^{b}$} \\
\hline & ADHD & $\mathrm{RD}$ & $\mathrm{ADHD}+\mathrm{RD}$ & Clinical controls & & \\
\hline $\mathrm{N}$ & 26 & 28 & 17 & 42 & & \\
\hline $\mathrm{N}$ male & 20 & 25 & 14 & 34 & & \\
\hline Age & $9.0(1.7)$ & $9.1(1.6)$ & $8.6(1.4)$ & $8.5(1.7)$ & & 0.190 \\
\hline SES & $33.6(10.2)$ & $36.6(12.3)$ & $38.5(13.4)$ & $38.3(10.2)$ & & 0.564 \\
\hline PPVT-3 & $103.0(10.5)$ & $99.7(11.1)$ & $99.8(11.2)$ & $103.9(10.0)$ & & 3.13 \\
\hline WJR numbers reversed & $100.8(17.2)$ & $91.7(12.3)$ & $95.2(13.1)$ & $99.0(15.8)$ & & $4.67^{*}$ \\
\hline CCPT overall index & $9.7(7.1)$ & $7.3(6.9)$ & $11.5(7.2)$ & $6.7(6.5)$ & 3.3 & 2.8 \\
\hline WJR sound blending & $93.0(12.2)$ & $85.3(11.0)$ & $83.0(8.3)$ & $89.6(10.8)$ & $7.27^{* *}$ & $11.15^{* * *}$ \\
\hline Time estimate 30 s passive & $29.8(18.2)$ & $31.0(25.5)$ & $30.5(19.5)$ & $29.8(21.8)$ & 0.01 & 0.044 \\
\hline Time estimate 30 s active & $62.8(37.9)$ & 74.7(88.9) & $63.2(36.9)$ & $76.2(72.0)$ & 0.66 & 0.87 \\
\hline CCPT duration estimate & $22.2(16.8)$ & $12.0(7.6)$ & $24.2(21.4)$ & $15.76(9.7)$ & $17.43^{* * *}$ & $16.49^{* * *}$ \\
\hline
\end{tabular}

${ }^{a}$ Covariates: age, SES, IQ (PPVT-3), working memory (WJR numbers reversed).

${ }^{b}$ Order (first or last) is covaried out of CCPT overall index and CCPT duration scores.

${ }^{*} p<.05$. $^{* *} p<.01$. $^{* * *} p<.001$.

with each other (ranging from .20 to $.32, p<.05$ ). None of the time perception variables were associated with any of the parent or teacher behavioral ratings.

\section{Analyses}

Each analysis employed a $2 \times 2$ (ADHD vs. RD) design. Each analysis employed the four core covariates. Because some authors argue against the use of covariates in clinical research (Miller \& Chapman, 2001), each analysis was repeated without the four core covariates. For all analyses, evaluation of assumptions of linearity, normality, multicollinearity or singularity, and homogeneity of variance-covariance matrices revealed no threat to multivariate analysis. To protect for Type 1 error, omnibus significance tests were evaluated (overall $F$ for the complete regression model). Then, the $F$ value for the main effects were examined, followed by post-hoc univariate $F$ tests only if both the omnibus $F$ test and the main effect $F$ test were significant beyond an alpha level of .05 . Table I presents the univariate $F$ test results for each covariate and criterion variable, for both covariate and noncovariate analyses.

\section{Hypothesis One: Group Differences on the CCPT}

To phrase our hypothesis positively, we predicted that the CCPT would distinguish between diagnostic groups. Unfortunately, our preliminary investigations showed that the CCPT was subject to a fatigue effect. To control for order effects, the CCPT was randomly assigned to first or last place in the 1.5 -hr battery. CCPT overall index scores were correlated with order of administration $(r=.28, p=.003)$, as were several other CCPT parameters (e.g., Hit Reaction time, $r=-.25$, omission errors, $r=.21, p s<.05)$.

On the Conners' CPT, an overall index score greater than 11 is considered to be a "failure" - an indication of attention problems (Conners, 1995). Chi-square analysis revealed that participants who completed the CCPT last in the battery were far more likely to fail it than those who did it first in the battery, $\chi^{2}(1)=10.4, p<.001$. Of those participants who failed the CCPT, $31 \%$ completed it first, whereas $68 \%$ completed it last. Said another way, $50 \%$ of the participants who did the CCPT last failed it, compared to $20 \%$ who did it first. All things being equal, a child was twice as likely to fail the CCPT if he or she completed it as the last task in a 1.5-hr battery. ADHD children were differentially impacted by this fatigue relative to non-ADHD participants $\left(\chi^{2}(1)=7.1, p<.01\right)$, but so too were RD children relative to non-RD children $\left(\chi^{2}(1)=6.6, p<.01\right)$. In a previous study, we did not find an order effect for the CCPT within a 1-hr battery (McGee et al., 2000). It would seem that a 1.5-hr battery is sufficiently taxing that CCPT performance degrades after the 1-hr mark. Because of this fatigue effect, we added order (first or last) to the covariate list when predicting CCPT parameters.

No main effects were detecting using any parameter of the CCPT. Controlling for order, there were no group differences on the CCPT. This is true even if one adds age, IQ, SES, and working memory as covariates. We did not replicate our own previous finding that CCPT overall index scores were greater among RD children (McGee et al., 2000). 


\section{Hypothesis Two: Group Differences on Time Perception}

Our second hypothesis was that time perception tasks would distinguish ADHD from RD youngsters and clinical controls. Two analyses were conducted: one on the 30-s estimates, and one on the CCPT duration estimate.

\section{Thirty-s Time Estimation}

The activity level (Active vs. Passive) of the 30-s estimate tasks served as a within-group factor, with diagnostic status as the between groups factor in a $2 \times 2$ ANOVA. No between group differences were found, with or without covariates. However, the within groups factor proved significant, $F(1,107)=44.8, p<.0001$. Children gave longer estimates to the active task $(M=70.8, S D=66)$ than the passive task $(M=30.2, S D=21)$, paired $t(110)=7.39$, $p<.0001$.

\section{CCPT Duration Estimate}

After they had completed the CCPT, participants estimated how long it took them to complete the standardduration CCPT. There was no association between CCPT order (first or last) and estimated time to complete the CCPT $(r=-.08, p=.39)$. There was also no association between CCPT time estimation and the difficulty of the CCPT for the child $(r=-.04$ with the CCPT overall index). Age was uncorrelated with CCPT time duration estimates.

The diagnostic groups differed significantly on retrospective time estimation. Even with all the covariates (including order) the overall model proved significant, $F(8,105)=2.4, p<.05$. A main effect for ADHD was found, $F(1,105)=17.43, p<.0001$. No main effect for RD status was detected, nor was the interaction significant. Without covariates, the main effect for ADHD remained significant, $F(1,105)=16.49, p<.0001$. Examination of means suggests that children with ADHD make far longer and more variable estimates of the time it took to complete the CCPT $(M=22.9 \mathrm{~min}, S D=18.4)$ compared to children without ADHD $(M=14.3 \mathrm{~min}$, $S D=9.0$ ). Moreover, non-ADHD participants were reasonably accurate in judging the duration of the CCPT (the actual duration is $14 \mathrm{~min}$ ). In contrast, the average ADHD child's estimate was 1.6 times longer than the actual duration. On average, children with ADHD judged the CCPT to be nearly 10 minutes longer than it is.

\section{Hypothesis Three: Group Differences on Auditory Phonological Awareness}

Our third hypothesis was that children with RD would perform more poorly than children without RD on measures of auditory phonological awareness, even when controlling for age, IQ, working memory, and SES. Using WJR Sound Blending scores as the criterion, a $2 \times 2$ $(\mathrm{ADHD} \times \mathrm{RD})$ ANCOVA was conducted. The overall model was significant, $F(7,105)=2.7, p=.01$, as was the test for main effects of RD status, $F(1,105)=7.27$, $p<.01$. Even without covariates, the main effect for RD status remained significant, $F(1,105)=11.15, p<.01$. Sound Blending scores were significantly lower among the RD group $(M=84.4, S D=10.0)$ than the non-RD group $(M=90.9, S D=11.28)$. There was no main effect for ADHD status, and no interaction. Even controlling for auditory working memory as well as other cognitive and social features, RD children struggled more with an auditory task of phonological awareness.

\section{Hypothesis Four: Comorbid Versus "Pure" Groups}

To interpret a DD design, one must examine group means to see if there are "additive" deficits in the comorbid group. Planned contrasts compared the mean of the comorbid group to the combined mean of the other "pure" diagnostic groups. As predicted, the comorbid group had a higher mean on CCPT time estimates $(M=25.5, S D=$ $21.5)$ relative to the "pure" groups $(M=16.9, S D=12.1)$, $t(105)=-2.1, p<.05$. Sound Blending means were relatively lower in the comorbid group, but this difference fell shy of significance $(p=.08)$. Thus, the mean differences tended to converge in the direction predicted by the "etiological subtype" hypothesis. No interaction effects were evident on any analysis, underscoring the distinctiveness of the disorders.

\section{DISCUSSION}

This study used a classical double-dissociation design contrasting ADHD and RD groups on measures of auditory phonological processes and time perception. Planned comparisons revealed that children with ADHD showed a significant impairment in retrospective time duration estimation, but no impairment on phonological processing. The RD group showed impairment in phonological processing, but no impairment in time duration estimation. Moreover, these process deficits are not simply a corollary of inattentiveness (for which all the participants 
were referred) or clinical status per se. The comorbid children had greater deficits relative to their diagnostic match on retrospective time estimation, and no interaction effects were found. Taken together, these data support the burgeoning research that suggests that RD and ADHD are two distinct disorders with separate cognitive profiles, and support the "etiological subtype" hypothesis over the "phenocopy" hypothesis (Purvis \& Tannock, 2000; Willcutt et al., 2001). Moreover, it ties in with the research emerging on the biogenetic markers for ADHD and RD as separate disorders with separate cognitive manifestations (e.g., Davis et al., 2001; Halperin et al., 1997; Willcutt, Pennington, \& DeFries, 2000).

It is worth noting that double-dissociation was obtained even under very conservative conditions. We found differences despite covarying out age, SES, IQ, and working memory, and using a nonspecific clinical control group. ADHD and RD participants were rigorously defined by stringent cutoffs, and borderline cases were assigned to the clinical control group. All these approaches decreased the likelihood of finding significant group differences, but served to increase the generalizability of the results.

Scores on the Conners' CPT did not distinguish ADHD children from the other clinical groups. Overall, these findings are similar to those of other studies in suggesting that CPT scores do not consistently identify attention problems in children deemed to have ADHD relative to other clinical groups (Corkum \& Siegel, 1993; Koelega, 1995; Trommer, Hoeppner, Lorder, \& Armstrong, 1988). However, the fatigue effect found in this study impaired the reliability and hence the validity of the CCPT as a measure of sustained attention. Moreover, the fatigue effects were more pronounced for RD and ADHD children. Perhaps ADHD and RD children are more sensitive to the effects of fatigue after a long battery of cognitive tasks, possibly consistent with their problems with cognitive processing. At minimum, our results suggest that the Conners' CPT ought not to be used at the end of a test battery longer than $1 \mathrm{hr}$.

This study provides more evidence of the specificity of phonological processing deficits to RD. It adds to the previous literature by showing that auditory (as opposed to visual) phonological deficits distinguish RD children from other clinical groups, even when controlling for auditory working memory-even when controlling for the method variance attributable to using auditory tasks as both predictor and criterion.

No group differences were found using the 30-s time estimates. Rather, age accounted for most of the variance in 30-s time estimates. Time production and time estimation tasks were uncorrelated, suggesting that they measure different processes. As in previous developmental studies, the more active and distracting the task the longer the time production (Zakay, 1992). This study used a time production method: future studies ought to use a time reproduction method. In time reproduction, the child is required to reproduce a time interval that has been specifically modeled for them. Barkley and his colleagues consider time reproduction methods to be more sensitive to diagnostic group differences (Barkley et al., 1997)

The most unusual and interesting finding of the study was discovering the retrospective exaggeration of ADHD children when judging how long the CCPT had taken. When asked "how long did that take?" of the CCPT, ADHD children gave more extreme estimates ("Oh! That took 3 hours!"). This finding underscores the temporal distortion problems found in ADHD (West et al., 2000). Childrens' time estimations become less accurate with increasing duration of the time interval to be perceived, and distortion decreases with age (Zakay, 1992). Barkley's theory emphasizes that ADHD childrens' time judgements will be developmentally delayed relative to agemates (Barkley, 1997a, 1997b, 1997c). Barkley, Murphy, and Bush (2001) found that ADHD adults made larger time estimates relative to normal controls, particularly at longer intervals. This study extends the previous research by showing time perception deficits relative to clinical controls, and controls for the influence of age. Most previous studies have examined time intervals no longer than $60 \mathrm{~s}$. This current study highlights temporal distortion of much longer time intervals, and underscores its relevance to everyday time judgments.

The nature of the CCPT task may also account for the findings. Several studies of smaller time durations in which focused attention was manipulated has suggested that focused attention increases the perception of the passage of time (Barkley et al., 2001). The CCPT requires focused attention-perhaps this burdened ADHD children sufficiently that their time perception distorted exponentially. Was the CCPT harder for ADHD children? Time estimates were not associated with success on the task, nor with the fatigue factor, suggesting that the tendency of ADHD children to overestimate its duration is not simply due to its relative difficulty for them.

The results of this study are partially consistent with Barkley's theory of ADHD (Barkley, 1997a, 1997b, 1997c) which emphasizes behavioral disinhibition and distorted time perception. Time production tasks did not differentiate ADHD from other clinical groups, but retrospective time estimation did. The tendency of the ADHD children to overestimate a retrospective time judgement may be due to behavioral disinhibition. Perhaps children with ADHD simply cannot inhibit an exaggerated or silly 
answer to the duration question. Anecdotally, ADHD children appeared to be answering in good faith-review of the behavioral observation notes did not suggest more deliberate flippancy in this group. Further study-perhaps quantifying the flippancy dimension-would be required to parse out how much distortion is due to temporal distortion and how much is due to disinhibited verbal responding.

This study has a number of limitations that need to be addressed in future research. The sample size of 113 clinical cases could be larger. Our results may not generalize to other measurement or diagnostic methods. For example, we did not define RD by the IQ-achievement discrepancy method recommended by DSM-IV. The CPT results may not extend to other versions, such as the AX version. Barkley (1997b) argues that the Inattentive Subtype of ADHD is qualitatively different than the Combined Type. As our ADHD sample was primarily the Combined Type, the results may not generalize to children with the Inattentive Only profile. We lack objective information on the reliability and validity of the time perception tasks. The study did not employ a "normal" control group, so we have no data on retrospective time estimation of the CCPT in nonclinical children. However, it is worth noting that non-ADHD clinical participants were nevertheless accurate in their judgements. On average, they estimated the CCPT to be 14 minutes long-which it is. We have no reason to believe that normal children would be less accurate at CCPT time estimation than clinical controls.

The finding that naturalistic time perception can be distorted in ADHD relative to other disorders invites further study. It would be interesting to explore the focused attention aspects of the task to be estimated. Would ADHD children overestimate the time spent on an interesting video game that was 14-minutes long? Do ADHD children overestimate the duration of daily tasks? We could ask ADHD children the duration of daily events (e.g., classes, recess, homework time). If ADHD children greatly overestimate the duration of "boring" tasks, this phenomenon may underlie the motivational deficits and lack of persistence found with this population. If a child "remembers" that yesterdays math homework took " 3 hours" (when it infact only took 14 minutes) - how would this perception impact his or her willingness to do homework today? Further research on short-term and long-term memory for task duration may help us tease out how temporal distortion, memory, and motivation intertwine in ADHD children.

\section{ACKNOWLEDGMENTS}

This study was supported by an Izaack Walton Killam Hospital for Children Charitable Foundation External
Grant (981017), Halifax, N.S., to the first author and a Social Sciences and Humanities Standard Research Grant (410-2000-0045) to the first three authors.

\section{REFERENCES}

Achenbach, T. (1991a). Manual for the Child Behavior Checklist/4-18 and 1991 Profile. Burlington, VT: University of Vermont.

Achenbach, T. (1991b). Manual for the Teacher's Report Form /4-18 and 1991 Profile. Burlington, VT: University of Vermont.

August, G., \& Garfinkel, B. (1990). Comorbidity of ADHD and reading disability among clinic-referred children. Journal of Abnormal Child Psychology, 18(1), 29-45.

Barkley, R. (1991). The ecological validity of laboratory and analogue assessment methods of ADHD symptoms. Journal of Abnormal Psychology, 19(2), 149-178.

Barkley, R. (1997a). Attention-deficit/hyperactivity disorder, selfregulation, and time: Toward a more comprehensive theory. Developmental and Behavioral Pediatrics, 18, 271-279.

Barkley, R. (1997b). ADHD and the nature of self control. New York: Guilford.

Barkley, R. (1997c). Behavioral inhibition, sustained attention, and executive functions: Constructing a unifying theory of ADHD. Psychological Bulletin, 121, 65-94.

Barkley, R. (1997d). Defiant children, 2nd ed. New York: Guilford.

Barkley, R., DuPaul, G., \& McMurray, M. (1990). Comprehensive evaluation of attention deficit disorder with and without hyperactivity as defined by research criteria. Journal of Consulting and Clinical Psychology, 58, 775-789.

Barkley, R., Edwards, G., Laneri, M., Fletcher, K., \& Metevia, L. (2001). Executive functioning, temporal discounting, and sense of time in adolescents with attention deficit hyperactivity disorder (ADHD) and oppositional defiant disorder (ODD). Journal of Abnormal Child Psychology, 29, 541-546.

Barkley, R., Grodzinsky, G., \& DuPaul, G. (1992). Frontal lobe functions in attention deficit disorder with and without hyperactivity: A review and research report. Journal of Abnormal Child Psychology, $20,163-188$

Barkley, R., Koplowitz, S., Anderson, T., \& McMurray, M. (1997). Sense of time in children with ADHD: Effects of duration, distraction, and stimulant medication. Journal of the International Neuropsychological Society, 3, 359-369.

Barkley, R., Murphy, K., \& Bush, T. (2001). Time perception and reproduction in young adults with attention deficit hyperactivity disorder. Neuropsychology, 15, 351-360.

Blishen, B. R., Carroll, W. K., \& Moore, C. (1987). The 1981 socioeconomic index for occupations in Canada. Canadian Review of Sociology and Anthropology, 24, 465-488.

Breier, J., Gray, L., Fletcher, J., Foorman, B., \& Klaas, P. (2002). Perception of speech and nonspeech stimuli by children with a reading disability and attention deficit hyperactivity disorder. Journal of Experimental Child Psychology, 82, 226250.

Brodeur, D., \& Pond, M. (2001). The development of selective attention in children with attention deficit hyperactivity disorder. Journal of Abnormal Child Psychology, 29, 229-239.

Castellanos, F., \& Tannock, R. (2002). Neuroscience of attentiondeficit/hyperactiviety disorder: The search for endophenotypes. National Review of Neuroscience, 3, 617-628.

Catts, H. (1989). Defining dyslexia as a developmental language disorder. Annals of Dyslexia, 39, 50-63.

Cohen, N., Vallance, D., Barwick, M., Im, N., Menna, R., Horodezky, N., et al. (2000). The interface between ADHD and language impairment: An examination of language, achievement, and cognitive processing. Journal of Child Psychology and Psychiatry, 41, 353362. 
Compton, D., DeFries, J., \& Olson, R. (2001). Are RAN- and phonological awareness-Deficits additive in children with reading disabilities? Dyslexia, 7, 125-149.

Conners, C. (1995). Conners' Continuous Performance Test. Toronto: Multi-Health Systems.

Conners, C. (1997). Conners' Rating Scales-Revised. Toronto: Multi-Health Systems.

Corkum, P., \& Siegel, L. (1993). Is the Continuous Performance Task a valuable research tool for use with children with attentiondeficit-hyperactivity disorder? Journal of Child Psychology and Psychiatry, 34, 1217-1239.

Davis, C., Gayan, J., Knopik, V., Smith, S., Cardon, L., Pennington, B., et al. (2001). Etiology of reading difficulties and rapid naming: The Colorado twin study of reading disability. Behavioral Genetics, $31,625-635$

Droit-Volet, S., \& Rattat, A. (1999). Are time and action dissociated in young children's time estimation? Cognitive Development, 14, 573-595.

Dunn, L., \& Dunn, L. (1997). The Peabody Picture Vocabulary Test-Third edition. Minnesota: American Guidance Service.

Eliason, M., \& Richman, L. (1987). The Continuous Performance Test in learning disabled and non disabled children. Journal of Reading Disabilities, 20, 614-619.

Felton, R., Wood, F., Brown, I., Campbell, S., \& Harter, M. (1987). Separate verbal memory and naming deficits in attention deficit disorder and reading disability. Brain and Language, 31, 171-184.

Fischer, M., Newby, R., \& Gordon, M. (1995). Who are the false negatives on continuous performance tests? Journal of Clinical Child Psychology, 24, 427-433.

Halperin, J., Newcorn, J., Koda, V., Pick, L., McKay, K., \& Knott, P. (1997). Noradrenergic mechanisms in ADHD children with and without reading disabilities: A replication and extension. Journal of the American Academy of Child and Adolescent Psychiatry, 36 , $1688-1697$

Kerns, K., McInerney, R., \& Wilde, N. (2001). Time reproduction, working memory, and behavioral inhibition in children with ADHD. Child Neuropsychology, 7, 21-31.

Kerns, K., \& Price, K. (2001). An investigation of prospective memory in children with ADHD. Child Neuropsychology, 7, 162-171.

Koelega, H. (1995). Is the continuous performance task useful in research with ADHD children? Comments on a review. Journal of Child Psychology and Psychiatry, 36, 1477-1485.

Koriath, U., Gualtieri, T., Van Bourgondien, M., Quade, D., \& Werry, J. (1985). Construct validity of clinical diagnosis in pediatric psychiatry: Relationship among measures. Journal of the American Academy of Child Psychiatry, 242, 429-436.

Korkman, M., \& Pesonen, A. (1994). A comparison of neuropsychological test profiles of children with attention deficit-hyperactivity disorder and/or learning disorder. Journal of Learning Disabilities, $6,383-392$.

Landau, M., Gross-Tsur, V., Auerbach, J., Van der Meere, J., \& Shalev, R. (1999). Attention-deficit hyperactivity disorder and developmental right-hemisphere syndrome: Congruence and incongruence of cognitive and behavioral aspects of attention. Journal of Child Neurology, 14, 299-303.

Losier, B., McGrath, P., \& Klein, R. (1996). Error patterns on the continuous performance test in non-medicated and medicated samples of children with and without ADHD: A meta-analytic review. Journal of Child Psychology and Psychiatry, 37, 971-987.

McBride-Chang, C. (1995). What is phonological awareness? Journal of Educational Psychology, 87, 179-192.

McGee, R., Clark, S., \& Symons, D. (2000). Does the Conners' Continuous Performance Test aid in ADHD diagnosis? Journal of Abnormal Child Psychology, 28, 415-424.
Miller, G., \& Chapman, J. (2001). Misunderstanding analysis of covariance. Journal of Abnormal Psychology, 110, 40-48.

Pennington, B., Groisser, D., \& Welsh, M. (1993). Contrasting cognitive deficits in attention deficit hyperactivity disorder versus reading disability. Developmental Psychology, 29, 511-523.

Pennington, B., \& Ozonoff, S. (1996). Executive functions and developmental psychopathology. Journal of Child Psychology and Psychiatry, 37, 51-87.

Purvis, K., \& Tannock, R. (2000). Phonological processing, not inhibitory control, differentiates ADHD and reading disability. Journal of the American Academy of Child and Adolescent Psychiatry, 39, 485-494.

Rucklidge, J., \& Tannock, R. (2002). Neuropsychological profiles of adolescents with ADHD: Effects of reading difficulties and gender. Journal of Child Psychology and Psychiatry, 43, 9881003.

Seidman, L., Biederman, J., Monuteaux, M., Doyle, A., \& Faraone, S. (2001). Learning disabilities and executive dysfunction in boys with attention deficit hyperactivity disorder. Neuropsychology, 15, 544-556.

Semrud-Clikeman, M., Biederman, J., Sprich-Buckminster, S., Lehman, B., Faraone, S., \& Norman, D. (1992). Comorbidity between ADHD and learning disability: A review and report in a clinically referred sample. Journal of American Academy of Child and Adolescent Psychiatry, 31, 439-448.

Shapiro, S., \& Herod, L. (1994). Combining visual and auditory tasks in the assessment of attention-deficit hyperactivity disorder In D. Routh (Ed.), Disruptive behavior disorders in childhood (pp. 87-107). New York: Plenum.

Smith, A., Taylor, E., Rogers, J., Newman, S., \& Rubia, K. (2002). Evidence for a pure time perception deficit in children with ADHD. Journal of Child Psychology and Psychiatry, 43, 529-542.

Stevens, J., Quittner, A., Zuckerman, J., \& Moore, S. (2002). Behavioral inhibition, self-regulation of motivation, and working memory in children with attention deficit hyperactivity disorder. Developmental Neuropsychology, 21, 117-139.

Tannock, R., Ickowicz, A., \& Schachar, R. (1995). Differential effects of methylphenidate on working memory in ADHD children with and without comorbid anxiety. Journal of the American Academy of Child and Adolescent Psychiatry, 34, 886-896.

Tarnowski, K., Prinz, R., \& Nay, S. (1986). Comparative analysis of attentional deficits in hyperactive and learning-disabled children. Journal of Abnormal Psychology, 95, 341-345.

Trommer, B., Hoeppner, J., Lorder, R., \& Armstrong, K. (1988). Pitfalls in the use of a continuous performance test as a diagnostic tool in attention deficit disorder. Journal of Developmental and Behavioral Pediatrics, 9, 339-346.

Willcutt, E., Pennington, B., Boada, R., Ogline, J., Tunick, R., Chhabildas, N., et al. (2001). A comparison of the cognitive deficits in reading disability and attention-deficit/hyperactivity disorder. Journal of Abnormal Psychology, 11, 157-172.

Willcutt, E., Pennington, B., \& DeFries, J. (2000). Twin study of the etiology of comorbidity between reading disability and attention-deficit/hyperactivity disorder. American Journal of Medical Genetics, 3, 293-301.

West, J., Douglas, G., Houghton, S., Lawrence, V., Whiting, K., \& Glasgow, K. (2000). Time perception in boys with attentiondeficit/hyperactivity disorder according to time duration, distraction and mode of presentation. Child Neuropsychology, 6, 241250

Woodcock, R., \& Johnson, M. (1989, 1990). Woodcock-Johnson Psychoeducational Battery-Revised. Chicago, IL: Riverside.

Zakay, D. (1992). The role of attention in children's time perception. Journal of Experimental Child Psychology, 54, 355-371. 